\title{
Combining non-specular X-ray scattering and X-ray absorption spectroscopy for the investigation of buried layers
}

\author{
D. Lützenkirchen-Hecht ${ }^{1}$, P. Keil', and R. Frahm ${ }^{1}$ \\ ${ }^{1}$ Fachbereich C-Experimentalphysik, Bergische Universität Wuppertal, Gaußstr. 20, 42097 Wuppertal, Germany. \\ ${ }^{2}$ Current address: Department of Interface Chemistry and Surface Engineering, Adhesion and Thin Films, \\ Max-Planck Institut für Eisenforschung GmbH, Max-Planck-Str. 1, 40237 Düsseldorf, Germany.
}

The atomic structure and the chemical composition of buried interfaces is of high interest for many applications e.g. in multilayered samples such as oxidized metals, optic or microelectronic devices. Conventional surface analytical techniques such as X-ray photoelectron spectroscopy (XPS), Auger-electron spectroscopy (AES) or ion scattering spectroscopy (ISS) have an extremely high surface sensitivity but are not suited for the non-destructive investigation of buried layers, where a sputter-depth profiling is required. Non-specular (diffuse) X-ray scattering is another method to obtain quantitative information of buried interfaces and multilayers [1-3]. In addition to the specular X-ray reflectivity, which provides information about the structures perpendicular to the surface of the sample (namely the surface and interface roughness, layer thickness and density), the diffuse X-ray scattering gives information on the in-plane and out-of-plane structure of the involved interfaces [3]. Especially lateral correlations are accessible using this technique, making it an ideal tool for the study of multilayered samples and liquid surfaces also (see, e.g. [2, 4]). As recently shown, the combination of grazing incidence non-specular X-ray scattering with X-ray absorption spectroscopy may give additional information such as the chemical valence and the local atomic environment of atoms which are located in surface and interface regions [5, 6]. Since the angular position of the Yoneda-peak is directly correlated with the density of the X-ray scattering material (e.g. [7]), different interfaces can be selected by the choice of the scattering angles. Therefore, by measuring the X-ray absorption fine structure for well defined incidence and exit angles, it is possible to study the atomic short range order and the chemical state of a chosen element in a chosen position inside a multilayered sample. In this contribution, we will demonstrate the capabilities of the new technique for the ex-situ investigation of a metallic $\mathrm{Cu}$-Au-bilayer prepared by subsequent sputtering of $\mathrm{Au}$ and $\mathrm{Cu}$ on a glass substrate.

The experiments described here were performed at the X-ray undulator beamline BW1 [8] using a $\mathrm{Si}(111)$ double crystal monochromator and $\mathrm{N}_{2}$ - and Ar-filled ionization chambers as detectors for incoming and scattered intensities. The incident X-ray beam was collimated vertically by a $120 \mu \mathrm{m}$ slit. The acceptance angle of the detector is equal to $0.04^{\circ}$ and $0.1^{\circ}$ for the specular und nonspecular case, respectively.

Non-specular grazing incidence $\mathrm{X}$-ray reflectivities measured from the $\mathrm{Cu}$-Au-bilayer on glass are presented in Fig. 1(a). In addition to the peak associated with the specular reflection of X-rays $\left(\alpha_{i}=\right.$ $\alpha_{\mathrm{f}}$ ), Yoneda-peaks associated with the reflection of X-rays from the outer air-Cu surface and the inner $\mathrm{Cu}-\mathrm{Au}$ interface are also visible. As can be seen from Fig. 1, these peaks shift slightly with energy according to the energy dependence of the real and the imaginary part of the complex refractive index $n(E)=1-\delta(E)-i * \beta(E)$ (see, e.g. ref. [5]). With a sufficiently large angular acceptance of the reflectivity detector of $\Delta \alpha_{\mathrm{f}}=0.1^{\circ}$, these variations can be overcome, so that it is possible to fix the detector at a certain exit angle and measure the variation of the Yoneda-peak intensity while scanning across the interesting absorption edges [5]. Related absorption scans measured at the $\mathrm{Cu}$ $\mathrm{K}$-edge are presented in Fig. 1(b). As can easily be seen, the reflection mode X-ray absorption fine structure changes dramatically both in amplitude and shape if the exit angle is changed from smaller to larger values. As can be seen in Fig. 1(b), both the near edge structure in the vicinity of the absorption edge (which is primarily related to the electronic structure of the sample under investigation) as well as the extended X-ray absorption fine structure (which is connected to the atomic short range order) are dramatically changed. 

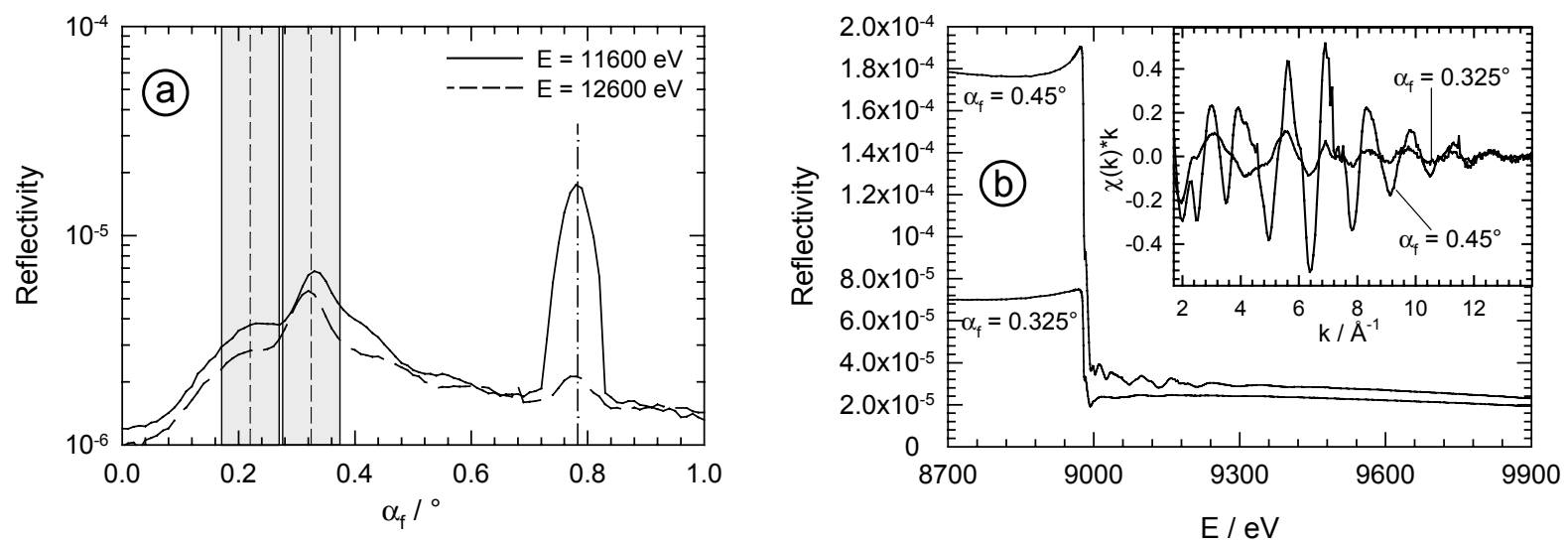

Fig. 1: (a) Comparison of detector-scans obtained from a $\mathrm{Cu} / \mathrm{Au}$-bilayer on a glass substrate for an incidence angle $\alpha_{\mathrm{i}}=0.79^{\circ}$ and photon energies $\mathrm{E}=11600 \mathrm{eV}$ and $\mathrm{E}=12600 \mathrm{eV}$. The exit angles $\alpha_{\mathrm{f}}$ chosen for non-specular reflection mode EXAFS experiments are indicated by dashed vertical lines in both graphs, and the slit integration of $0.1^{\circ}$ around these angles is denoted by grey stripes. In addition, the specular reflection peaks at $\alpha_{\mathrm{i}}=\alpha_{\mathrm{f}}$ are shown by dash-dotted vertical lines. (b) Non-specular reflection mode EXAFS experiments at the $\mathrm{Cu}$ K-edge for an incidence angle $\alpha_{\mathrm{i}}=0.7^{\circ}$ and exit angles $\alpha_{\mathrm{f}}=0.325^{\circ}$ and $\alpha_{\mathrm{f}}=0.45^{\circ}$ as indicated. The acceptance angle of the detector was $\Delta \alpha_{\mathrm{f}}=0.1^{\circ}$. In the insert, the kweighted reflectivity fine structure extracted from these spectra is presented.

Similar to the conventional transmission EXAFS data analysis, the EXAFS was extracted from the raw reflectivity data by means of the subtraction of a smooth background and a transformation from the photon energy scale to a photoelectron wave number scale. In the insert of Fig. 1(b), k-weighted reflectivity fine structure oscillations $\chi(\mathrm{k}) * \mathrm{k}$ are presented. While both the near edge structure and the EXAFS of the data measured for $\alpha_{\mathrm{f}}=0.45^{\circ}$ are similar to that of metallic copper, the data measured for $\alpha_{\mathrm{f}}=0.325^{\circ}$ are akin to copper oxide. Having in mind that the two different Yonedapeaks are belonging to the outer $\mathrm{Cu}$-air-surface and the inner $\mathrm{Cu}$-Au-interface, this finding clearly implies that the outer surface of the bilayer is oxidized by the laboratory air, in agreement with previous experiments [5]. However, the short range order structure of the $\mathrm{Cu}$ in the inner $\mathrm{Cu}-\mathrm{Au}$ interface is very similar to that of polycrystalline metallic copper. In addition, no gold absorption edge is visible for the smaller exit angle, while the fine structure measured for the larger exit angle (related to the inner interface) clearly reveals fine structures of metallic gold. These findings suggest that no gold is present at the outer surface, and that alloying of the two elements at the inner interface is not present in substantial amounts [5]. In conclusion, we have shown that the combination of grazing incidence non-specular X-ray scattering and X-ray absorption spectroscopy allows non-destructive investigations of buried interfaces. More specifically, in the case of a $\mathrm{Cu}-\mathrm{Au}$ thin film bilayer system, it allows the separate investigation of the atomic short range order structure of the outer air- $\mathrm{Cu}$ surface and the inner $\mathrm{Cu}-\mathrm{Au}$ interface using both the $\mathrm{Cu}$ and the $\mathrm{Au}$ atoms as local probes in each position in the bilayer, as described in more detail in ref. [9].

This project was funded by the MWF (Nordrhein-Westfalen). Additional support by HASYLAB is gratefully acknowledged.

\section{References}

[1] S.K. Sinha. J. Phys. III (France) 4 (1994) 1543.

[2] V. Holy, U. Pietsch and T. Baumbach. Springer Tracts in Mod. Phys. 149 (1999).

[3] S.K. Sinha, E.B. Sirota, S. Garoff and H.B. Stanley. Phys. Rev. B 38 (1988) 2297.

[4] M. Tolan. Springer Tracts in Mod. Phys. 148 (1999).

[5] P. Keil, D. Lützenkirchen-Hecht, R. Frahm. Europhys. Lett. 71 (2005) 77.

[6] P. Keil, D. Lützenkirchen-Hecht, R. Frahm. Physica B 357 (2005) 1.

[7] J.-L. Cao, A. Solbach, U. Klemradt, T. Weirich, J. Mayer, U. Böttger, U. Ellerkmann, P.J. Schorn, P. Gerber and R. Waser. J. Amer. Ceram. Soc. 89 (2006) 1321.

[8] R. Frahm, J. Weigelt, G. Meyer and G. Materlik. Rev. Sci. Instrum. 66 (1995) 1677.

[9] D. Lützenkirchen-Hecht, P. Keil, R. Frahm, Surf. Sci. (2006), in press. 